\title{
Measurement of the Light Antiquark Flavor Asymmetry in the Nucleon Sea
}

\author{
E.A. Hawker ${ }^{j}$, T.C. Awes ${ }^{i}$, M.E. Beddo ${ }^{h}$, C.N. Brown ${ }^{c}$, J.D. Bush ${ }^{a}$, T.A. Carey ${ }^{f}$, T.H. Chang ${ }^{h}$, W.E. Cooper ${ }^{c}$, \\ C.A. Gagliardi ${ }^{j}$, G.T. Garvey ${ }^{f}$, D.F. Geesaman ${ }^{b}$, X.C. He ${ }^{d}$, L.D. Isenhower ${ }^{a}$, S.B. Kaufman ${ }^{b}$, D.M. Kaplan ${ }^{e}$, \\ P.N. Kirk ${ }^{g}$, D.D. Koetke ${ }^{k}$, G. Kyle ${ }^{h}$, D.M. Lee ${ }^{f}$, W.M. Lee ${ }^{d}$, M.J. Leitch ${ }^{f}$, N. Makins ${ }^{6}$, P.L. McGaughey ${ }^{f}$, \\ J.M. $\operatorname{Moss}^{f}$, B.A. Mueller ${ }^{b}$, P.M. Nord ${ }^{k}$, B.K. Park ${ }^{f}$, V. Papavassiliou ${ }^{h}$, J.C. Peng ${ }^{f}$, G. Petitt ${ }^{d}$, P.E. Reimer ${ }^{f}$, \\ M.E. Sadler ${ }^{a}$, J. Selden ${ }^{h}$, P.W. Stankus ${ }^{i}$, W.E. Sondheim ${ }^{f}$, T.N. Thompson ${ }^{f}$, R.S. Towell ${ }^{a}$, R.E. Tribble ${ }^{j}$, \\ M.A. Vasiliev插, Y.C. Wang ${ }^{g}$, Z.F. Wang ${ }^{g}$, J.C. Webb ${ }^{h}$, J.L. Willis ${ }^{a}$, D.K. Wise ${ }^{a}$, G.R. Young ${ }^{i}$
}

$$
\text { (FNAL E866/NuSea Collaboration) }
$$

\footnotetext{
${ }^{a}$ Abilene Christian University, Abilene, TX 79699

${ }^{b}$ Argonne National Laboratory, Argonne, IL 60439

${ }^{c}$ Fermi National Accelerator Laboratory, Batavia, IL 60510

${ }^{d}$ Georgia State University, Atlanta, GA 30303

${ }^{e}$ Illinois Institute of Technology, Chicago, IL 60616

${ }^{f}$ Los Alamos National Laboratory, Los Alamos, NM 87545

${ }^{g}$ Louisiana State University, Baton Rouge, LA 70803

${ }^{h}$ New Mexico State University, Las Cruces, NM, 88003

${ }^{i}$ Oak Ridge National Laboratory, Oak Ridge, TN 37831

${ }^{j}$ Texas A 83 M University, College Station, TX 77843

${ }^{k}$ Valparaiso University, Valparaiso, IN 46383
}

(February 7, 2008)

\begin{abstract}
A precise measurement of the ratio of Drell-Yan yields from an $800 \mathrm{GeV} / \mathrm{c}$ proton beam incident on hydrogen and deuterium targets is reported. Over 140,000 Drell-Yan muon pairs with dimuon mass $M_{\mu^{+} \mu^{-}} \geq 4.5 \mathrm{GeV} / \mathrm{c}^{2}$ were recorded. From these data, the ratio of anti-down $(\bar{d})$ to anti-up $(\bar{u})$ quark distributions in the proton sea is determined over a wide range in Bjorken- $x$. A strong $x$ dependence is observed in the ratio $\bar{d} / \bar{u}$, showing substantial enhancement of $\bar{d}$ with respect to $\bar{u}$ for $x<0.2$. This result is in fair agreement with recent parton distribution parameterizations of the sea. For $x>0.2$, the observed $\bar{d} / \bar{u}$ ratio is much nearer unity than given by the parameterizations.
\end{abstract}

13.85.Qk; 14.20.Dh; 24.85.+p; 14.65.Bt

No known symmetry requires equality of the $\bar{d}$ and $\bar{u}$ distributions in the proton. Until recently it had been generally assumed that $\bar{d}(x)=\bar{u}(x)$ for lack of experimental evidence to the contrary. This assumption may be evaluated by use of the expression

$$
\int_{0}^{1}\left[F_{2}^{p}(x)-F_{2}^{n}(x)\right] \frac{d x}{x}=\frac{1}{3}-\frac{2}{3} \int_{0}^{1}\left[\bar{d}_{p}(x)-\bar{u}_{p}(x)\right] \mathrm{d} x .
$$

Here $F_{2}^{p}(x)$ and $F_{2}^{n}(x)$ are the proton and neutron inelastic structure functions, and $\bar{d}_{p}(x)$ and $\bar{u}_{p}(x)$ are the anti-down and anti-up quark distributions in the proton sea as a function of Bjorken- $x$. Equation 1 requires the assumption of charge symmetry between the proton and neutron (i.e. $u_{p}=d_{n}, \bar{u}_{p}=\bar{d}_{n}$, etc.). If the nucleon sea is flavor symmetric in the light quarks, the value of the integral on the left is $1 / 3$, a result referred to as the Gottfried Sum Rule (GSR) [1]. In 1991 the New Muon Collaboration (NMC) at CERN presented evidence that the GSR is violated, based on deep inelastic muon scattering data from hydrogen $(p)$ and deuterium $(d)$. They reported a final value of $\int_{0}^{1}\left[F_{2}^{p}(x)-F_{2}^{n}(x)\right] \frac{d x}{x}=0.235 \pm 0.026$ [2], which implies that

$$
\int_{0}^{1}\left[\bar{d}_{p}(x)-\bar{u}_{p}(x)\right] d x=0.147 \pm 0.039
$$

a considerable excess of $\bar{d}_{p}$ relative to $\bar{u}_{p}$. This result has been adopted in the most current parameterizations of the parton distributions in the nucleon 3., 4 .

Following publication of the NMC result, the use of the Drell-Yan process [5] was suggested [6] as a means by which the light antiquark content of the proton could be more directly probed. This was first done by the Fermilab E772 collaboration. They compared the production of Drell-Yan muon pairs from isoscalar targets to that from a neutron rich target. This measurement sets constraints on the non-equality of $\bar{u}$ and $\bar{d}$ in the range $0.04 \leq x \leq$ 0.27 [7]. Later, the CERN experiment NA51 [8] carried out a comparison of the Drell-Yan muon pair yield from hydrogen and deuterium at a single value of $x$ using a $450 \mathrm{GeV} / \mathrm{c}$ proton beam and found

$$
\left.\frac{\bar{u}_{p}}{\bar{d}_{p}}\right|_{\langle x\rangle=0.18}=0.51 \pm 0.04 \pm 0.05 .
$$

A recent review by Kumano [9] presents an extensive discussion of the existing literature on the flavor asymmetry of the antiquark sea. 
Fermilab Experiment 866 (E866) measured the DrellYan muon pair yield from $800 \mathrm{GeV} / \mathrm{c}$ proton bombardment of liquid deuterium and hydrogen targets. From these data, $\bar{d} / \bar{u}$ and $\bar{d}-\bar{u}$ in the proton over the range $0.020<x<0.345$ are extracted. A significant difference between the $\bar{d}$ and $\bar{u}$ distributions is found.

E866 used a 3-dipole magnet spectrometer 10 employed in previous experiments (E605, E772, and E789), modified by the addition of new drift chambers and hodoscopes with larger acceptance at the first tracking station. Other improvements to the spectrometer included a programmable trigger system [11] and a VME-based data acquisition system. An $800 \mathrm{GeV} / \mathrm{c}$ extracted proton beam with up to $2 \times 10^{12}$ protons per $20 \mathrm{~s}$ spill bombarded one of three identical $50.8 \mathrm{~cm}$ long cylindrical target flasks containing either liquid hydrogen, liquid deuterium or vacuum. After passing through the target, the remaining beam was intercepted by a copper beam dump located inside the second dipole magnet. The beam dump was followed by a 13.4 interaction length absorber wall of copper, carbon and polyethylene which blocked the entire aperture of the magnet. This absorber wall removed hadrons produced in the target and the dump, ensuring that only muons traversed the spectrometer's detectors. The detection system consisted of four tracking stations and a momentum analyzing magnet. The spectrometer's acceptance as a function of $p_{T}$, the transverse momentum of the dimuon pair, was reasonable to $3.0 \mathrm{GeV} / \mathrm{c}$, with some acceptance to $5.0 \mathrm{GeV} / \mathrm{c}$.

The targets alternated between hydrogen and deuterium every five beam spills with a single spill collected on the empty flask at each target change. The targets were 3.54 and $8.14 \mathrm{~g} / \mathrm{cm}^{2}$ thick, corresponding to 7 and $15 \%$ of an interaction length for hydrogen and deuterium, respectively. Beam intensity was monitored by secondary-emission detectors, an ion chamber and quarter-wave RF cavities. Two four-element scintillator telescopes viewing the targets at nearly $90^{\circ}$ monitored the luminosity, beam duty factor and data acquisition live time. The trigger required a pair of triple hodoscope coincidences having the topology of a muon pair from the target. Typically 70 triggers per second were recorded with an electronic live time above $98 \%$. An integrated flux of $1.3 \times 10^{17}$ protons was delivered.

Over 330,000 Drell-Yan events were recorded, using three different spectrometer settings which focused low, intermediate and high mass muon pairs. The data collected with the low and intermediate mass settings have systematic effects of a few percent which require additional study. The data from the high mass setting are relatively free from these effects due to the greatly reduced rates in the tracking chambers. Therefore, this Letter presents only the results from the high mass setting, with over 140,000 Drell-Yan events.

In calculating the Drell-Yan yields, a small correction (averaging $0.2 \%$ ) for random coincidences between two

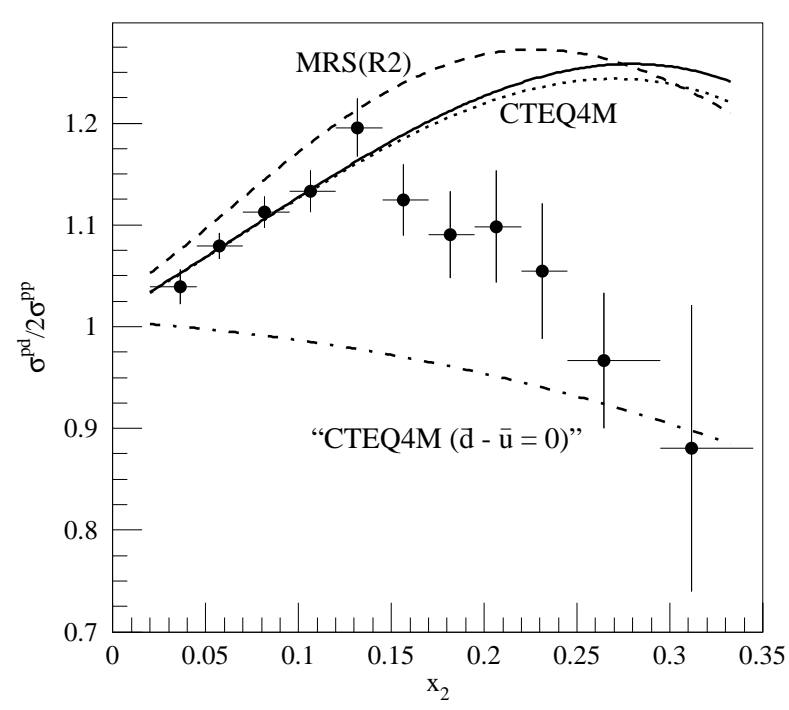

FIG. 1. The ratio $\sigma^{p d} / 2 \sigma^{p p}$ of Drell-Yan cross sections $v s$. $x_{2}$. Shown are next-to-leading order calculations, weighted by acceptance, of the Drell-Yan cross section ratio using the CTEQ4M (solid) and MRS(R2) (dashed) parton distributions. Also shown is a leading order calculation of the cross section ratio using CTEQ4M (dotted). In the lower CTEQ4M curve $\bar{d}-\bar{u}$ has been set to 0 as described in the text. The errors are statistical only. An additional $1 \%$ systematic uncertainty is common to all points.

unrelated, oppositely charged muons was made. This correction was evaluated by studying the observed rates of same charge muon pairs. A background rate of approximately $8 \%$ from the target flask and beam line windows was measured using the evacuated flask and was subtracted. Given the identical geometry of the deuterium and hydrogen targets and the slight difference in the average interaction point due to beam attenuation in the target, acceptance differences between the two targets were shown by a Monte Carlo simulation of the spectrometer to be very small. The measured cross section ratio was corrected for differences in beam attenuation in the targets, target density and a small hydrogen contamination in the deuterium target. Additionally, the high luminosity caused a small, rate dependent inefficiency. This led to a correction of $1.2 \%$ in the cross section ratio. The systematic error in the ratio of yields from the two targets is dominated by the uncertainties in the rate dependence $( \pm 0.6 \%)$, hydrogen contamination in the deuterium target $( \pm 0.2 \%)$ and beam attenuation $( \pm 0.2 \%)$. All other contributions, including the production of muon pairs from secondary hadron reinteraction, are small. The total systematic error in the cross section ratio is less than $\pm 1 \%$.

The resulting ratio of the Drell-Yan cross section per nucleon for $p+d$ to that for $p+p$ is shown in Fig. 1 and Table [1] as a function of $x_{2}$ [12],13], the momentum fraction (Bjorken- $x$ ) of the target quark in the parton model. 
TABLE I. Ratio of deuterium to hydrogen cross sections per nucleon vs. $x_{2}$. The average of the kinematic variables for each bin is also tabulated. Note that $\left\langle x_{1}\right\rangle=\left\langle x_{F}\right\rangle+\left\langle x_{2}\right\rangle$. The errors are statistical only. An additional $1 \%$ systematic uncertainty is common to all points.

\begin{tabular}{ccccc}
\hline \hline & & $\begin{array}{c}\left\langle p_{T}\right\rangle \\
(\mathrm{GeV} / \mathrm{c})\end{array}$ & $\begin{array}{c}\left\langle M_{\mu}+\mu^{-}\right\rangle \\
\left(\mathrm{GeV} / \mathrm{c}^{2}\right)\end{array}$ & $\sigma^{p d} / 2 \sigma^{p p}$ \\
\hline$\left.x_{2}\right\rangle$ & $\left\langle x_{\mathrm{F}}\right\rangle$ & 0.92 & 5.5 & $1.039 \pm 0.017$ \\
0.036 & 0.537 & 1.03 & 6.5 & $1.079 \pm 0.013$ \\
0.057 & 0.441 & 1.13 & 7.4 & $1.113 \pm 0.015$ \\
0.106 & 0.369 & 1.18 & 7.9 & $1.133 \pm 0.020$ \\
0.132 & 0.294 & 1.21 & 8.5 & $1.196 \pm 0.029$ \\
0.156 & 0.220 & 1.21 & 9.3 & $1.124 \pm 0.035$ \\
0.182 & 0.192 & 1.20 & 9.9 & $1.091 \pm 0.043$ \\
0.207 & 0.166 & 1.19 & 10.6 & $1.098 \pm 0.055$ \\
0.231 & 0.134 & 1.18 & 11.1 & $1.055 \pm 0.067$ \\
0.264 & 0.095 & 1.18 & 11.8 & $0.967 \pm 0.067$ \\
0.312 & 0.044 & 1.12 & 12.8 & $0.881 \pm 0.141$ \\
\hline \hline
\end{tabular}

(The Bjorken- $x$ of the beam parton is denoted by $x_{1}$.) Muon pairs with mass, $M_{\mu^{+} \mu^{-}}$, below $4.5 \mathrm{GeV} / \mathrm{c}^{2}$ or between 9.0 and $10.7 \mathrm{GeV} / \mathrm{c}^{2}$ were removed to eliminate contributions from the $J / \psi$ and $\Upsilon$ resonance families. The data clearly show that the Drell-Yan cross section per nucleon for $p+d$ exceeds $p+p$ over an appreciable range in $x_{2}$. Figure 1 also shows the predictions for next-to-leading order calculations [14] of the cross section ratio, weighted by the E866 spectrometer's acceptance, using the CTEQ4M [3] and MRS(R2) [4] parton distributions. The lower curve shows the predicted ratio for a modified CTEQ4M parton distribution which maintains the parameterization for $\bar{d}_{p}+\bar{u}_{p}$ but sets $\bar{d}_{p}-\bar{u}_{p}=0$. The data are in reasonable agreement with the unmodified CTEQ4M and the MRS(R2) predictions for $x_{2}<0.15$. It is clear that $\bar{d}_{p} \neq \bar{u}_{p}$ in this range. Above $x_{2}=0.15$ the data lie well below both parameterizations.

The acceptance of the spectrometer was largest for $x_{\mathrm{F}}=x_{1}-x_{2}>0$. In this kinematic regime the Drell-Yan cross section is dominated by the annihilation of a beam quark with a target antiquark. This fact, coupled with the assumption of charge symmetry between the neutron and proton and the assumption that the deuteron parton distributions can be expressed as the sum of the proton and neutron distributions, yields a simple approximate form of the Drell-Yan cross section ratio,

$$
\left.\frac{\sigma^{p d}}{2 \sigma^{p p}}\right|_{x_{1} \gg x_{2}} \approx \frac{1}{2} \frac{\left(1+\frac{1}{4} \frac{d_{1}}{u_{1}}\right)}{\left(1+\frac{1}{4} \frac{d_{1}}{u_{1}} \frac{\bar{d}_{2}}{\bar{u}_{2}}\right)}\left(1+\frac{\bar{d}_{2}}{\bar{u}_{2}}\right) .
$$

The subscripts 1 and 2 denote that the parton distributions in the proton as functions of $x_{1}$ and $x_{2}$, respectively. In the case that $\bar{d}=\bar{u}$, the ratio is 1 . This equation illustrates the sensitivity of the Drell-Yan measurement to $\bar{d} / \bar{u}$ and is valid only for $x_{1} \gg x_{2}$. It does, however, imply an excess of $\bar{d}$ with respect to $\bar{u}$ for the data. Esti-

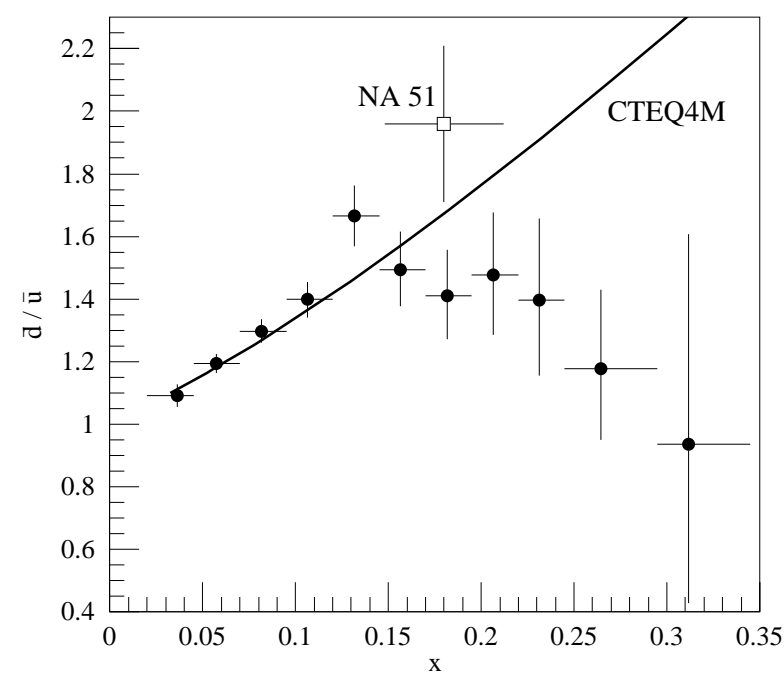

FIG. 2. The ratio of $\bar{d} / \bar{u}$ in the proton as a function of $x$ extracted from the Fermilab E866 cross section ratio. The curve is from the CTEQ4M parton distributions. The error bars indicate statistical errors only. An additional systematic uncertainty of \pm 0.032 is not shown. The result from NA51 is also plotted as an open box.

mates of the nuclear effects in deuterium are significantly less than statistical errors shown in Fig. 11 115.

Some of the data, especially at higher $x_{2}$, do not satisfy the $x_{1} \gg x_{2}$ criterion of Eq. \&. Consequently, $\bar{d} / \bar{u}$ was extracted iteratively by calculating the leading order Drell-Yan cross section ratio using a set of parton distribution functions as input and adjusting $\bar{d} / \bar{u}$ until the calculated cross section ratio agreed with the measured value. In this procedure, the values for the $\bar{d}+\bar{u}$, valence and heavy quark distributions given by the global fits [e.g., CTEQ4M and MRS(R2)] were assumed to be correct. In the beam proton, when $x_{1} \leq 0.345$, the $\bar{d} / \bar{u}$ distribution was assumed to be the same as in the target proton. For $x_{1}>0.345$, a constant value of 1 for $\bar{d} / \bar{u}$ was used. Varying the high- $x_{1}$ value of $\bar{d} / \bar{u}$ produced almost no change in the low $x_{2}$ bins, and less than a $3 \%$ change in the highest $x_{2}$ bin. This procedure was followed using both the CTEQ4M and MRS(R2) parameterizations and negligible differences were seen. The extracted $\bar{d} / \bar{u}$ ratio is shown in Fig. 2 along with the prediction made by CTEQ4M. A qualitative feature of the data, not seen in either parameterization, is the rapid decrease towards unity of the $\bar{d} / \bar{u}$ ratio beyond $x=0.2$. At $x=0.18$, the extracted $\bar{d} / \bar{u}$ ratio is somewhat smaller than the value obtained by NA51. Although the average value of $Q^{2}$ $\left(M_{\mu^{+} \mu^{-}}^{2}\right)$ is different for the two data sets, the change in $\bar{d} / \bar{u}$ predicted by the parton distributions due to $Q^{2}$ evolution is small.

To address the GSR violation observed by NMC, the extracted $\bar{d} / \bar{u}$ ratio is used together with the CTEQ4M value of $\bar{d}+\bar{u}$ to obtain $\bar{d}-\bar{u}$. (Nearly identical results 


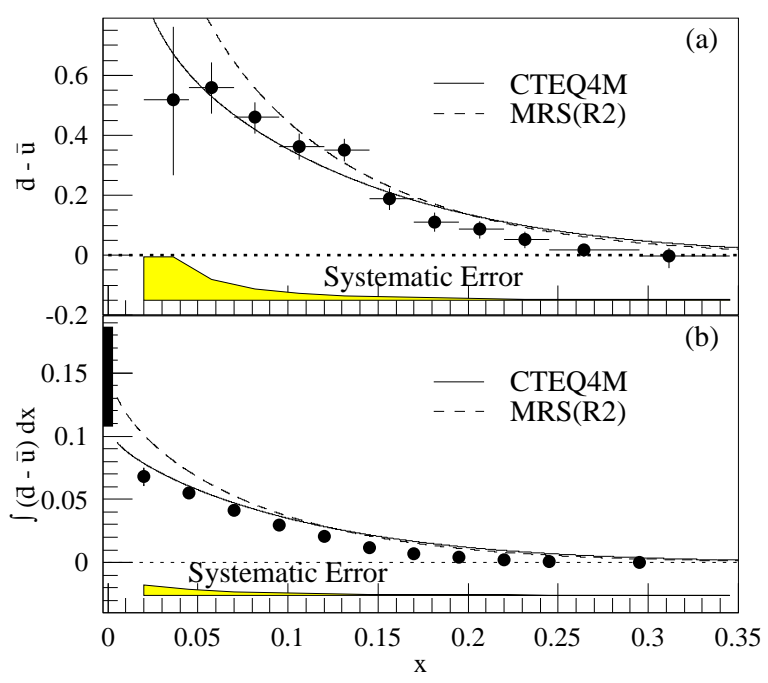

FIG. 3. Fermilab E866 values for (a) $\bar{d}-\bar{u}$ and (b) $\int_{x}^{0.345}(\bar{d}-\bar{u}) d x^{\prime}$ in the proton versus $x$. The curves represent the corresponding values obtained by the CTEQ4M (solid) and MRS(R2) (dashed) parameterizations. The bar at $0.147 \pm 0.039$ on the left axis in (b) shows the result obtained by NMC for the integral from 0 to 1 .

are obtained if MRS(R2) is used instead of CTEQ4M.) Since each bin in $x$ has a different average $Q^{2}, \bar{d}-\bar{u}$ was scaled to a common $Q$ value of $7.35 \mathrm{GeV} / \mathrm{c}$, the average for the entire data set. Based on this, the integral of $\bar{d}-\bar{u}$ between $x^{\min }$ and 0.345 is calculated. Both $\bar{d}-\bar{u}$ and $\int_{x_{\min }}^{0.345}(\bar{d}-\bar{u}) d x$ are shown in Fig. 3. The integral reaches a value of $0.068 \pm 0.007$ (stat) \pm 0.008 (syst) at $x^{\text {min }}=0.02$. This may be compared with the CTEQ4M and MRS(R2) parameterizations which have values of 0.076 and 0.100 , respectively, for the integral over the same region. Over the range $10^{-4}<x<1$, CTEQ4M gives a value of 0.108 for the integral, and MRS(R2) gives 0.160 . Above $x=0.345$, it is unlikely there are significant contributions to the $\bar{d}-\bar{u}$ integral since the sea is relatively small in this region. Both CTEQ4M and MRS(R2) find this region contributes less than 0.002 to the total integral. It is clear, however, that significant contributions to the integral arise in the unmeasured region below $x=0.02$.

Such a large $\bar{d} / \bar{u}$ asymmetry cannot arise from perturbative effects [16]. Most parameterizations of the parton distribution functions (e.g. CTEQ, MRS) simply assume a shape for $\bar{d}-\bar{u}$ that accommodates the NMC and NA51 results. It has been suggested 17.18 that including the effects of virtual mesons can account for the observed asymmetry and this appears 1922 to be at least qualitatively correct.

In summary, this Letter reports a measurement of the Drell-Yan cross section ratio per nucleon of $p+d$ to $p+p$. From this measurement the asymmetry of the light quark sea in the proton is extracted as a function of $x$. A feature of the present result is the reduction in $\bar{d} / \bar{u}$ for $x>0.2$. The current data are in qualitative agreement with NA51, but with a smaller $\bar{d} / \bar{u}$ ratio at the single value of $x$ which they measured. Over the range of $x$ covered in this experiment $(0.020<x<0.345)$, the integral of $\bar{d}-\bar{u}$ reaches a value of $0.068 \pm 0.007$ (stat) \pm 0.008 (syst). However, contributions to the integral arise from the unmeasured region below $x=0.020$.

We would like to thank the Fermilab Particle Physics, Beams and Computing Divisions for their assistance in performing this experiment. This work was supported in part by the U.S. Department of Energy.

* Present address: University of Illinois, Urbana, IL 61801.

$\dagger \quad$ Also with University of Texas, Austin, TX 78712.

¥ On leave from Kurchatov Institute, Moscow 123182, Russia.

[1] K. Gottfried, Phys. Rev. Lett. 18, 1174 (1967).

[2] P. Amaudruz et al., Phys. Rev. Lett. 662712 (1991); M. Arneodo et al., Phys. Rev. D 50, R1 (1994).

[3] H.L. Lai et al., Phys. Rev. D 55, 1280 (1997).

[4] A.D. Martin, R.G.Roberts and W.J. Stirling, Phys. Lett. B 387, 419 (1996).

[5] S.D. Drell and T.M. Yan, Ann. Phys. (NY) 66, 578 (1971).

[6] S.D. Ellis and W.J. Stirling, Phys. Lett. B 256, 258 (1991).

[7] P.L. McGaughey et al., Phys. Rev. Lett. 69, 1726 (1992).

[8] A. Baldit et al., Phys. Lett. B 332, 244 (1994).

[9] S. Kumano, Phys. Reports. (to be published).

[10] G. Moreno et al., Phys. Rev. D 43, 2815 (1991).

[11] C.A. Gagliardi et al., Nucl. Instrum. Methods (submitted).

[12] We define $x_{F}=\left(x_{1}-x_{2}\right)=2 p_{l} / \sqrt{s}$, where $p_{l}$ is the longitudinal momenta of the muon pair in the center of mass and $s$ is the total center of mass energy squared.

[13] Distributions of kinematic variables within bins may be obtained by contacting C.N. Brown at chuckb@fnal.gov.

[14] The code necessary to calculate the next-to-leading order cross section ratio was provided by W.K. Tung of CTEQ.

[15] D.M. Alde et al., Phys. Rev. Lett. 64, 2479 (1990).

[16] D.A. Ross and C.T. Sachrajda, Nucl. Phys. B 149, 497 (1979).

[17] A.W. Thomas, Phys. Lett. B 126, 97 (1983); A. Signal, A.W. Schreiber, and A.W. Thomas, Mod. Phys. Lett. A 6, 271 (1991).

[18] E.M. Henley and G.A. Miller, Phys. Lett. B 251, 453 (1990).

[19] W-Y.P. Hwang, J. Speth and G.E. Brown, Z. Phys. A 339, 383 (1991).

[20] S. Kumano, Phys. Rev. D 43, 3067 (1991); 43, 59 (1991); S. Kumano and J.T. Londergan, ibid. 44, 717 (1991)

[21] E.J. Eichten, I. Hinchcliff, and C. Quigg, Phys. Rev. D 45, 2269 (1992); 47, R747 (1993).

[22] A. Szczurek, M. Ericson, H. Holtzman, and J. Speth, Nucl. Phys. A 596, 397 (1996). 